\title{
Detection of inter-harmonics based on spectrum leakage
}

\author{
Jingxiang Zeng, Xingong Cheng, Xiju Zong,Yimei Li \\ University of Jinan, China \\ zengjingxx@126.com
}

Keywords: inter-harmonic; Fast Fourier Transform (FFT); spectrum leakage; window function

\begin{abstract}
With the extensive application of a large number of nonlinear electrical components, harmonic and inter-harmonic components in power system become more and more complex. The harmonics, inter-harmonic and fundamental wave are in the superposition state. The traditional harmonic detection method is Fast Fourier Transform (FFT), which can realize the accurate detection and analysis of integer harmonics. But in the measurement of the harmonic,the actual signal detected should be by discretization using the sampling signal, which caused the phenomenon of spectrum leakage and fence phenomenon. However, since inter-harmonic is relatively small, the harmonic wave and fundamental wave become the noise of detecting inter-harmonic. These problems become a huge obstacle to the amplitude, frequency and phase of the accurate detection of inter-harmonic. In this paper, the spectrum leakage phenomenon is discussed.Based on the characteristics of the harmonic and fundamental wave, the improved algorithm is proposed. The method is based on simple transformation of Fast Fourier Transform (FFT) and combine respective characteristics of harmonics and inter-harmonic to solve the problem of measuring harmonic and inter-harmonic interference and achieve accurate detection of inter-harmonic. The method is effective in combination with the three point method, so it is more competitive with respect to other methods. The method has the advantages of simple operation, fast calculation and high accuracy, which can provide an effective method for the harmonic detection of power quality analysis.
\end{abstract}

\section{Introduction}

With the use of a large number of power electronic devices and nonlinear components, harmonic pollution in power system becomes more and more serious and complex, which not only exists in harmonics, but also in the presence of inter-harmonics [1][2]. The existence of these harmonics seriously affects the safety and economic operation of power system [3].Incandescent lamps flicker which is caused by inter-harmonics have an effect on operating personnel and voltage fluctuations can damage transformers, instrumentation and Relay protection misoperation. Therefore, it is necessary to detect the inter-harmonics accurately in order to provide a good basis for the management of the inter-harmonics.

In the past, the detection methods of inter-harmonics is Fast Fourier Transform (FFT), least square method, wavelet transform method, spectral estimation method and Hilbert HuangTransform(HHT) method[9]. FFT algorithm can accurately measure and calculate harmonics, but for inter-harmonic detection, the traditional FFT will produce spectral leakage and fence effect which will effect the detection of inter-harmonics, such as the phase angle, amplitude of the inter-harmonic and inter-harmonic frequency. Using the interpolation algorithm can solve the problem of the fence, but can not eliminate measurement errors due to the spectral leakage. Using window algorithm can reduce the spectrum leakage errors, but need construct window function to make spectrum analysis become more complex. By using the least square method and independent component analysis method, the fundamental wave and harmonic can be easily detected, inter-harmonics can be separated, but the real-time performance is not good and there are some limitations in the detection of inter-harmonics [4]. The wavelet transform has the advantages of good local performance and it is not restricted by the whole period and synchronous sampling. However, under the influence of wavelet base, large amount of computation and hardware implementation become more difficult [5]. Using the method of 
spectrum estimation can real reflect the signal information but the amplitude and phase of the harmonic can not be detected[6]. By using Hilbert Huang Transform(HHT), resolution ability and local adaptability are higher and exact analysis can be achieved easily, but it can easily appear the phenomenon of endpoint effect and mode mixing [7][8].

In this paper, we first analyze the spectrum leakage phenomenon in the FFT algorithm and the interference between fundamental wave, harmonic and inter-harmonic[10] .An improved algorithm is proposed to eliminate the interference between the fundamental and harmonic currents. This algorithm changes the FFT algorithm a bit. The characteristic of window function is used to eliminate the interference of fundamental wave and harmonics in the measurement of harmonics to realize the accurate measurement of the inter-harmonic.The method is effective in combination with the three point method, so it is more competitive

\section{The spectrum leakage phenomenon of FFT algorithm}

In the theory, the FFT is the transformation of the measured signal for the whole period, but in the practical operation, we need to apply FFT only in one or several periods of the measured signal.

First,there is an infinite harmonic signal $x_{\mathrm{m}}(t)$, the signal amplitude is $A_{m}$, frequency is $\omega_{m}$ and phase angle is $\theta$, as shown in equation (1).

$$
x_{m}(t)=A_{m} e^{j}\left(\omega_{m} t+\theta\right)
$$

The the result of FFT for this signal is a line at the $\omega_{m}$.

We assume a window function with a width of $T$ and deal with this function by FFT.The results are shown in the equation 2 and 3.

$$
\begin{aligned}
W_{T}(t) & = \begin{cases}1 & 0<t<T \\
0 & \text { Other }\end{cases} \\
W_{T}(\omega) & =\frac{\sin (\omega T / 2)}{\omega / 2} \exp \left(-j \frac{\omega T}{2}\right)
\end{aligned}
$$

Multiply these two functions. The multiplication operation in the time domain is equivalent to the convolution operation in the frequency domain.The results are shown in the equation 4 and 5.

$$
\begin{aligned}
& \overline{x_{m}}(t)=x_{m}(t) W_{T}(t) \\
& \overline{x_{m}}=A_{m} \frac{\sin \left(\frac{\omega-\omega_{m}}{2} T\right)}{\frac{\omega-\omega_{m}}{2}} \exp (j \theta) \exp \left(-j \frac{\omega-\omega_{m}}{2} T\right)
\end{aligned}
$$

By using the equation 5, we can find the relation between the amplitude and frequency of the continuous function obtained by FFT.We can make a graph by MATLAB, as shown in Figure 1.

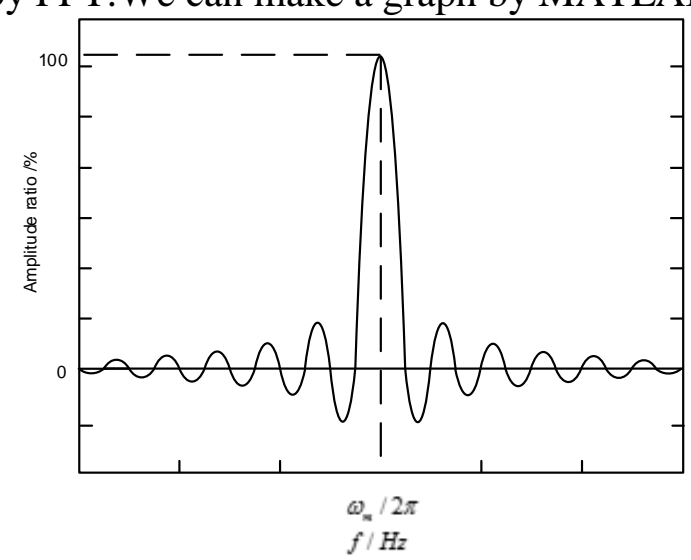

Figure 1. Single waveform spectrum

The above mentioned is the traditional calculation method for the continuous signal.In the practical engineering application, our data sources are discrete.In the numerical analysis, it is 
equivalent to the discrete processing of continuous signals. $\mathrm{n}$ is the sampling points of a period for $\omega=\frac{2 \pi n}{T}$.You can use the following equation(6) to represent the spectrum ( $k$ is integer).

$$
\overline{x_{m}}(n)=A_{m} N \frac{\sin ((n-k) \pi)}{(n-k) 2 \pi} \exp (j \theta) \exp (-j(n-k) \pi)
$$

The following conclusions can be drawn from the equation. Only when $n$ and $k$ meet $n=k$, equation will have value, when not, the value is equal to 0.So in the use of FFT to measure the integer harmonics there is a great advantage. $\mathrm{K}$ indicates the number of harmonics measured.

$$
\left\{\begin{array}{cc}
\overline{x_{m}}(n)=\frac{A_{m} N \exp (j \theta)}{2} & n=k \\
\overline{x_{m}}(n)=0 & n \neq k
\end{array}\right.
$$

When $k$ is not equal to an integer, it is relatively complex. We assume $k=k_{l}+r, k_{l}$ is an integral part, $r$ is a fractional part.

$$
\begin{aligned}
& \overline{x_{m}}(n)=A_{m} N \frac{\sin \left(\left(n-k_{1}\right) \pi-r \pi\right)}{2 \pi\left(n-k_{1}-r\right)} \exp (j \theta) \exp \left(-j\left(n-k_{1}-r\right) \pi\right) \\
& \overline{x_{m}}(n)=\frac{M}{\left(n-k_{1}-r\right)}=\frac{M}{t}
\end{aligned}
$$

Equation (9) is the result of simplified equation (8).

$$
\begin{aligned}
& t=n-k_{1}-r \\
& M=\frac{-A_{m} N \sin (r \pi) \exp (j(\theta+r \pi))}{2 \pi}
\end{aligned}
$$

The following conclusions can be drawn from the above deduction.With the increase of $n$, the $n-k_{1}-r$ decreases.Spectrum line corresponding to the maximum amplitude is at $n=k_{1}$ or $n=k_{1}+1$. With the increase of $n-k-r$, the amplitude of the spectral line is damped by $1 / t$.

We use Matlab to prove spectrum leakage phenomenon.In the FFT theory, the signal is measured in the whole time domain.However, in practical engineering, we need to cut off the measured signal.The value of a period of time is used to approximate the signal in the whole time domain.Under the premise of satisfying the sampling theorem, the sampling time period is not an integer multiple of the sampling time.This will be the phenomenon of spectrum leakage.Using MATLAB simulation, the graphics are shown as follows.

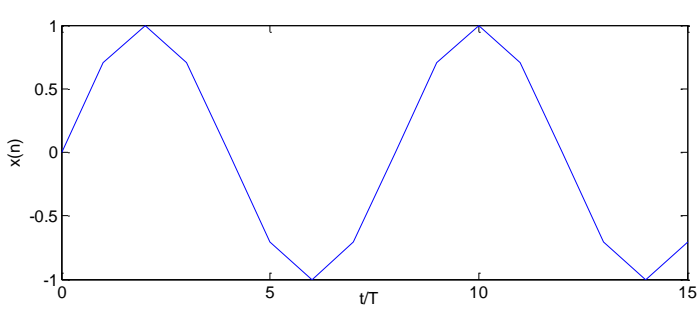

Figure 2 The signal be measured

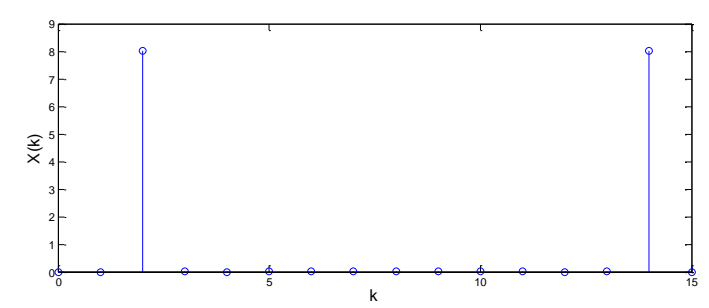

Figure 3 FFT analysis under ideal condition

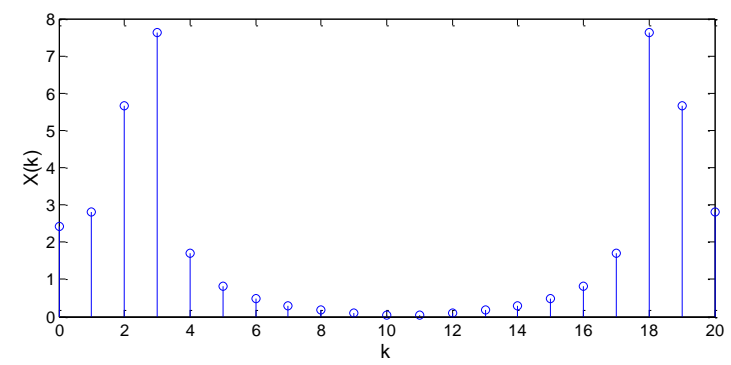

Figure 4 Spectrum leakage phenomenon

Compared with the above two spectrum of figure 3 and figure 4.We can clearly see the spectrum leakage phenomenon. This phenomenon is caused by the periodic extension of asynchronous sampling data. 


\section{Spectrum interference between harmonics and inter-harmonics}

In the system, there are fundamental, harmonic and inter-harmonic. They interact with each other, which has become a huge obstacle to accurate detection of inter-harmonics. Below we explain through mathematical derivation:

We have a set of signals $x(t)$

$$
x(t)=\sum_{p=1}^{p} A_{p} \cos \left(2 \pi f_{p} t+\varphi_{p}\right)
$$

This represents the number of harmonics or inter-harmonics. The $A$ indicates the magnitude of the signal.The f represents frequency and $\psi$ represents phase angle.Each index indicates the number of harmonics and inter-harmonics.

The equation (13) is obtained by the discretization of continuous signals.

$$
x[k]=\sum_{p=1}^{p} A_{p} \cos \left(2 \pi f_{p} \cdot k \cdot \Delta t+\varphi_{p}\right)
$$

Where the range of $\mathrm{k}$ is $0 \leqslant k \leqslant M N-1$.Under the condition that the measured signal is truncated by the rectangular window. The $\mathrm{M}$ represents the number of fundamental frequencies that are included in the rectangular window. The $\mathrm{N}$ represents the sampling points in a fundamental frequency.Use FFT to deal with the signal $x[k]$ and the results are shown in the equation (14).

$$
X\left(e^{j \Omega}\right)=\sum_{k=0}^{M N-1} x[k] e^{-j \Omega k}
$$

$\Omega=2 \pi f \Delta t$ DTF transform is performed on the equation, the results as shown in the equation (15).

$$
X[n]=\sum_{k=0}^{M N-1} x[k] e^{-j 2 m i k /(M N)}
$$

We can come to a conclusion, the results are shown in the equation (16).

$$
X[n]=\left.X\left(e^{j \Omega}\right)\right|_{\Omega=2 \pi n /(M N)}=X_{N}\left(e^{j 2 \pi n /(M N)}\right)
$$

We take absolute value to the result of DFT and the results are shown in the equation (17).

$$
\begin{aligned}
& X[n]=\left.X\left(e^{j \Omega}\right)\right|_{\Omega=2 \pi l /(M N)}=X\left(e^{j 2 m / /(M N)}\right) \\
& =\sum_{p=1}^{p} \frac{A_{p}}{2} e^{j\left[\varphi_{p}-\pi\left(n-f_{p} T_{W}\right) \frac{M N-1}{M N}\right]} \cdot \frac{\sin \pi\left(n-f_{p} \cdot T_{W}\right)}{\sin \frac{\pi}{M N}\left(n-f_{p} \cdot T_{W}\right)}
\end{aligned}
$$

Where the range of $\mathrm{n}$ is $0 \leqslant n \leqslant M N / 2-1$

$$
X\left(e^{j \Omega}\right)=\sum_{p=1}^{p} \frac{A_{p}}{2} e^{\left[\varphi_{p}-(M N-1)\left(\Omega-\Omega_{p}\right) / 2\right]} \cdot \frac{\sin \left[M N \cdot\left(\Omega-\Omega_{p}\right) / 2\right]}{M N \cdot\left(\Omega-\Omega_{p}\right) / 2}
$$

$X\left(e^{j \Omega}\right)$ The value at any frequency is the superposition of the corresponding value of each component at this point in the signal.Spectral interference between the various components will occur.

\section{Inter-harmonic detection method}

We find in the system, only the fundamental wave and harmonic components of the system are analyzed by using the method of the rectangular window with $\mathrm{M}$ fundamental wave period in the actual operation. The continuous signal is processed by DFT to obtain the spectrum of the main lobe and side lobe.In the system, only the fundamental and harmonic wave is analyzed and the spectrum shown in figure 5 can be obtained. 


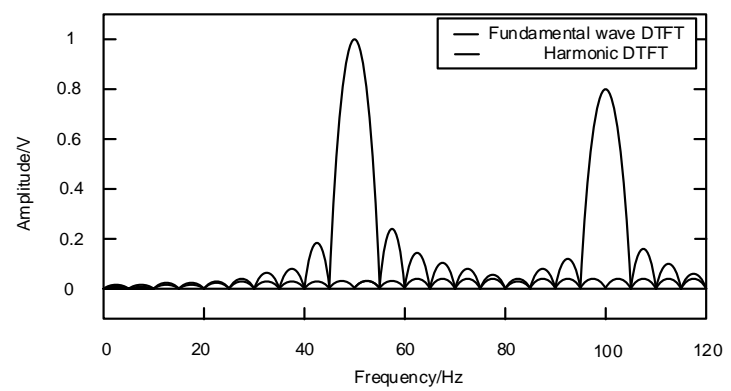

Figure 5. Fundamental wave and harmonic DTFT spectrum

Just because of the fundamental wave and harmonics in the system, the amplitude of the integer multiple of the side lobe width is 0 .The widths of the main lobe and side lobe are obtained by $2 \pi / N$.

When the system is in the presence of fundamental wave, harmonic and inter-harmonic, the spectrum leakage will occur.There is a numerical value at the frequency point of the integral multiple of the side lobe width.There are fundamental wave, harmonic and inter-harmonic currents in the system, and the following spectrum figure (6) can be obtained.

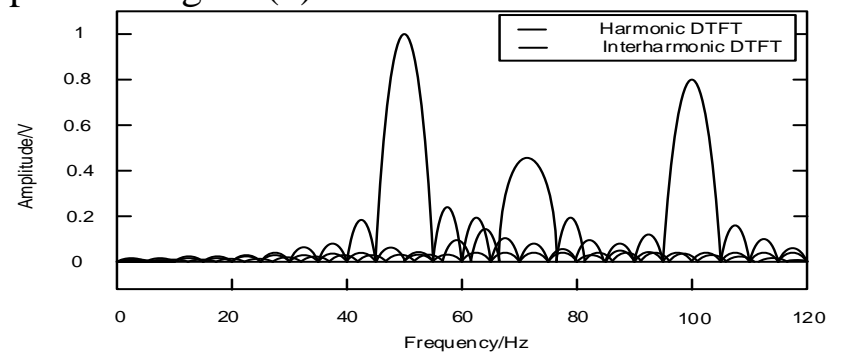

Figure 6. Harmonic and inter-harmonic DTFT spectrum

This value is just the numerical value of the inter-harmonic itself.We can use this value to detect the inter-harmonics.So as to eliminate the interference between the fundamental wave and harmonic.

\subsection{Using FFT to calculate the single inter-harmonic}

The system is the combination of fundamental wave, harmonic, and single frequency inter-harmonics. We can calculate the value of the special point in the spectrum figure.The special point is the end point of the side lobe. This value can well describes the characteristic information of the inter-harmonics. It can be found from the discrete spectrum amplitude of the maximum two lines: The peak value of the main lobe is far greater than the peak value of the side lobe.Assume that the two largest spectral lines are $n=k_{1}$ and $n=k_{1}+1$.

By using these two values, we can determine the corresponding parameters of the inter-harmonics. The amplitudes of these two lines are compared. Ratio is a and the results are shown in the equation (19).

$$
\begin{aligned}
& a=\left|\frac{\overline{x_{m}}\left(k_{1}\right)}{\overline{x_{m}}\left(k_{1}+1\right)}\right|=\left|\frac{M /(-r)}{M /(1-r)}\right|=\frac{1-r}{r} \\
& r=\frac{1}{1+a}
\end{aligned}
$$

We can draw the following conclusions.

$$
\begin{aligned}
& A_{m}=\left|\frac{2 \pi M}{N \sin (r \pi)}\right|=\left|\frac{2 \pi \overline{x_{m}}\left(k_{1}\right) r}{N \sin (r \pi)}\right| \\
& \theta=\operatorname{phase}\left(\overline{x_{m}}\left(k_{1}\right)\right)-\pi r
\end{aligned}
$$

\subsection{Selection of different window function}

In the signal processing, for window function there will be a lot of options.All kinds of window function contrast is shown below.

Form 1. The basic parameters of window function 


\begin{tabular}{|c|c|c|c|}
\hline $\begin{array}{c}\text { Type of window } \\
\text { function }\end{array}$ & $\begin{array}{c}\text { Side lobe peak } \\
a_{n} / d B\end{array}$ & $\begin{array}{c}\text { width of the transition zone } \\
\text { Approximate value }\end{array}$ & $\begin{array}{c}\text { Minimum stop } \\
\text { band attenuation } \\
a_{s} / d B\end{array}$ \\
\hline Rectangular & -13 & $4 \pi / N$ & -21 \\
\hline Bartlett window & -25 & $8 \pi / N$ & -25 \\
\hline Hanning window & -31 & $8 \pi / N$ & -44 \\
\hline Hamming window & -41 & $8 \pi / N$ & -53 \\
\hline Blackman & -57 & $12 \pi / N$ & -74 \\
\hline Kaiser window & -57 & $10 \pi / N$ & -80 \\
\hline
\end{tabular}

The following conclusions can be drawn from the table. For different window functions, the attenuation speed and the side lobe width are different. The attenuation of the rectangular window is relatively slow, but it can meet the requirement of finding the two maximum.Small side lobe width is the biggest advantage of rectangular window.There are many inter-harmonics in the system, which can play a huge role.In practical engineering, the rectangular window algorithm is simple.Because of these advantages, we choose a rectangular window as a tool in the detection of inter-harmonics.

\section{Conclusion}

This paper presents a method of detecting inter-harmonics by using improved FFT algorithm.By taking the value of the side lobe endpoint in the spectrum diagram, the interference of fundamental wave and harmonic wave to inter-harmonic will be avoided. To compare the characters of various window functions, the advantage of the rectangular window in the algorithm is proved in realizing the detection of inter-harmonics. The correctness of the algorithm is verified by simulation.The improved algorithm has the advantages of easy realization, simple analysis and high precision.This is an accurate and practical inter-harmonic analysis algorithm.It provides an effective method for harmonic detection and analysis in power system.

\section{References}

[1] Nguyen T T. Parametric harmonic analysis [of power systems] [C]. Generation, Transmission and Distribution, IEEE Proceedings, 1997, 144(1): 21-25.

[2] Yacamini R. Power system harmonics. IV. interharmonics[J]. Power Engineering Journal, 1996, 10(4):,185-193.

[3] Davis E J,.Emanuel A E. Harmonic pollution metering: theoretical considerations[J]. IEEE Transactions on Power Delivery, 2000, 15(1): 19-23.

[4] Gunther E W. Inter-harmonics in power systems[C] IEEE Power Engineering Society Summer Meeting Vancouver, BC, Canada, 2001, 2: 813-817.

[5] Hyvarinen A. Fast and robust fixed-point algorithms for independent component analysis[J]. IEEE Transactions on Neural Networks, 1999, 10(3): 626-634.

[6] Hui Xue and Rengang Yang.Precise algorithms for harmonic analysis based on FFT algorithm[J].China Agriculture University, Beijing 100083, China, 2002, 12: 106-110.

[7] Zhang Fusheng,Geng Zhongxing,Ge Yaozhong.FFT algorithm with high accuracy for harmonic analysis in power system[J].Proceedings of the CSEE.1999,19(3): 63-66.

[8] Li Gengyin,Chen Zhiye,Ning Yu.Two modified algorithms for Fast Fouried Transform[J].Automation of Electric Power Systems.1997,21(12): 37-40. 
[9] El-Habrouk and M. K. Darwish.Design and Implementation of Modified Fourier Analysis Harmonic Current Computation Technique for Power Active Filters using DSPS[J].IEE,Proc-Electr.Power Appl.2001,148(1):21-27.

[10] Zhu T X. Exact harmonics/interharmonics calculation using adaptive window width[J]. IEEE Transactions on Power Delivery, 2007, 47(1): 2279-2288. 